\title{
Fade Mitigation for Air-to-Ground Communication in Aeronautical Telemetry
}

\author{
Manisha Bhatt \\ Assistant Professor \\ Department of Electronics \& \\ Instrumentation \\ IEC College of Engineering \\ and Technology, Greater \\ Noida, India
}

\author{
Gopal Patel \\ Scientist ' $E$ ' \\ Defence Electronics \\ Applications Laboratory (DEAL) \\ Dehradun, India
}

\author{
Mukesh Pathela \\ Assistant Professor \\ Department of Electronics \& \\ communication Engineering \\ Dehradun Institute of \\ Technology, Dehradun, India
}

\begin{abstract}
Aeronautical Telemetry links were comprised of a transmit antenna mounted on the underside of fuselage and a fixed ground station equipped with a tracking antenna. Air-toground channel for this telemetry link can be estimated as line of sight propagation. But there is link loss observed at ground control station for certain instant of time. Possible causes of link loss in this case are due to aircraft maneuvering and fading. So analysis of various fade mitigation techniques as well as bit error rate (BER) and signal to noise (SNR) ratio performance comparison between selection diversity, equal gain combining and maximal ratio combining scheme is done. Finally bit error rate performance comparison curve between maximal ratio scheme (MRC) and Alamouti dual antenna transmission scheme is plotted, in order to find out the best scheme for fade mitigation, among all the above mentioned schemes.
\end{abstract}

\section{General Terms}

Aeronautical Telemetry, Air-to-ground communication, Fade mitigation.

\section{Keywords}

Aircraft maneuvering, Aeronautical Telemetry, Alamouti code, Diversity, Fade mitigation, SC, MRC, EGC.

\section{INTRODUCTION}

Whenever government want to buy an airplane, it has to be fully tested in order to ensure its reliability and safety. An air force test pilot flies this airplane at extreme speed to push it to the limits and this data is analyzed by ground crew in order to monitor its safety and performance.

Historically air plane uses only one antenna, which is mounted below the fuselage to send data to the ground crews. Air-to-ground telemetry links consists of a transmit antenna mounted on the underside of fuselage \& a fixed ground control station equipped with a tracking antenna. The tracking system continuously tracks the aircraft and provides the angular position of the aircraft with respect to the ground control station. This tracking system is so accurate in its performance, so that air-to- ground telemetry link can be estimated as line of sight (LOS) propagation. In spite of this LOS propagation the performance of link is not optimum as there is signal drop observed at certain instant of time [1]. In order to acquire the missing data, jet had to be refuelled, the equipment reassembled and mission reflow, which cost a lot of money.

Compromise with safety and reliability is not acceptable in this case, but we can reduce expenses in testing by finding out the solution to get rid of those data drop outs, so we don't have to refuel and retest. So in order to sort out this problem, entire work is subdivided into following three phases. In first phase detailed analysis of possible causes of signal loss has been carried out. Possible cause of signal loss is due to aircraft maneuvering and fading.

As aircraft maneuvers, LOS propagation path get blocked, because fuselage come in between the transmit antenna and receive antenna. In order to mitigate this problem, two antenna system transmitting the same signal is used. One antenna is mounted on the top of fuselage, while the second antenna is mounted on the bottom of the fuselage.

In second phase detailed analysis of various fade mitigation techniques has been done. In last phase bit error rate (BER) and signal to noise (SNR) ratio performance comparison between selection diversity, equal gain combining (EGC) and maximal ratio combining (MRC) scheme is done. Finally bit error rate performance comparison curve between maximal ratio scheme (MRC) and Alamouti dual antenna transmission scheme is plotted.

\section{POSSIBLE CAUSES OF SIGNAL LOSS}

\subsection{Due to Aircraft Maneuvering}

The air-to-ground telemetry link is a key component of the test and evaluation of airborne systems such as aircraft and missiles. Historically, aircraft links were comprised of a transmit antenna mounted on the underside of the fuselage and a fixed ground station equipped with a tracking antenna. Although air-to-ground channel can be estimated as LOS (Line of sight) propagation certain aircraft maneuvers can block the required line-of-sight propagation path. Experimental results show that aircraft during maneuvering can introduce shadowing on the transmitted signal for the airto-ground communication link. The shadowing can be up to $28 \mathrm{~dB}$ for forced turning maneuvering at $5.7 \mathrm{GHz}$, and can be up to $9.5 \mathrm{~dB}$ for the slight maneuvering while in a linear flight route [1]. These levels of shadowing are enough to cause a total lost in link synchronization and connection. Therefore, fading mitigation techniques such as multiple antenna communication are required to improve the air-to-ground links.

\subsection{Due to Fading}

For aeronautical telemetry the signal in air-to-ground link experiences strong line-of-sight (LOS) path in addition to diffracted and reflected paths from the surface of the aircraft. 
This is more likely in C-band where the wavelength is about 6 $\mathrm{cm}$. There are several papers available in the literature that supports the strong scattering from the aircraft body [2], [3]. Thus, the total field at the receiver $(\mathrm{Rx})$ antenna is the coherent addition of the field at the Rx. Due to difference in the path length, the phase of the multipath components arriving at the $\mathrm{Rx}$ will be different resulting in the fluctuation in the signals, here we exclude the possibility of scattering from the nearby ground vegetation.

Due to relative motion between the ground control station and the aircraft, each multipath wave experiences an apparent shift in frequency. This shift in received signal frequency due to motion is called Doppler shift, and is directly proportional to the velocity and direction of motion of the aircraft with respect to the direction of the arrival of the received multipath wave [4].The channel can be termed as slow-fading channel if the spectral dispersion due to Doppler effect is negligible as compared to spectral width of the baseband. In terms of coherence time, a channel is Slow fading if

$$
\text { Ts } \ll<\mathrm{Tc}
$$

Where Ts is Symbol duration and Tc is Coherence time. For our case of aeronautical telemetry, chip rate is $9.6 \mathrm{Mcps}$. Hence symbol duration is $\mathrm{Ts}=104 \mathrm{~ns}$. Coherence time Tc is related to Doppler spread as

$$
\mathrm{Tc}=0.423 / \mathrm{fm}
$$

Where $\mathrm{fm}$ is the Doppler spread and it is the maximum Doppler spread computed as $\mathrm{fm}=\mathrm{v} / \lambda$. This gives $\mathrm{Tc}=128 \mu \mathrm{s}$. Hence we see that equation (2) is satisfied. Thus, we can conclude that channel is slow-fading channel. Therefore, our channel falls in the category of slow fading channel with envelop statistics as Ricean distribution.

\section{Analysis of Fade Mitigation Techniques}

There are several fade mitigation techniques. One obvious solution is to employ two antenna system transmitting the same signal. One antenna is mounted on the top of fuselage, while the second antenna is mounted on the bottom of the fuselage. Whenever the line-of-sight (LOS) propagation from the bottom antenna to the ground station is blocked, link is maintained by the LOS propagation from the top antenna.

As the two antennas are transmitting the same signal and these two antennas are separated in space, the two signal received at the ground control station have difference in their phases, which causes constructive as well as destructive interference resulting in undesirable gain pattern.

Second solution is to use different carrier frequencies for the two antennas. While simple, this solution requires twice the bandwidth and can claim the use of two ground station antennas. The current challenges with spectrum allocations for telemetry are well-known solutions that require more bandwidth is unworkable. Another solution is to equip the aircraft with a steerable antenna and a mechanism for pointing the transmitted signal directly at the ground station. This family of solutions requires sophisticated antenna technology to implement electronically steerable antennas and a provision for computing the steering vectors: either an uplink signal used to correct errors in the steering vector or onboard computation based on time space position and the ground station location.

Time Diversity repeatedly transmits information at time spacing that exceed the coherence time of the channel, so that multiple repetitions of the signal will be received with independent fading conditions, thereby providing for diversity. But it is in effective when the channel is slowly varying or slow fading is there, which is present in the present scenario.

In most scattering environments, space diversity is a practical, effective and, hence, a widely applied technique for reducing the effect of multipath fading. The classical approach is to use multiple antennas at the receiver and perform combining or selection and switching in order to improve the quality of the received signal.

\subsection{Diversity Combining Methods}

Diversity combining [5] is used to overcome the problem of fading in radio channels and utilizes the fact that the signals arriving at different locations fade at different rates. A system employing a diversity combiner uses signals induced on various antennas placed a few wavelengths apart at different locations and combines these signals in one of many ways. In general, increasing the number of antennas results in more reduction in channel fading. However, in practice, 10-20 antennas seem to provide satisfactory results [6]

The collection of independently fading signal branches can then be combined in a variety of ways to improve the received SNR. Since the chance of having two deep fades from two uncorrelated signals at any instant is rare, combining them can reduce the effect of the fades. The three most prevalent space diversity-combining techniques are selection combining (SC), equal gain combining (EGC), and maximal ratio combining (MRC). In selection combining method (SC), the strongest signal branch is selected as shown in figure 1. While in maximal ratio combining method (MRC), the diversity branches are weighted for maximum $\mathrm{SNR}$ as can be seen in figure 2 .

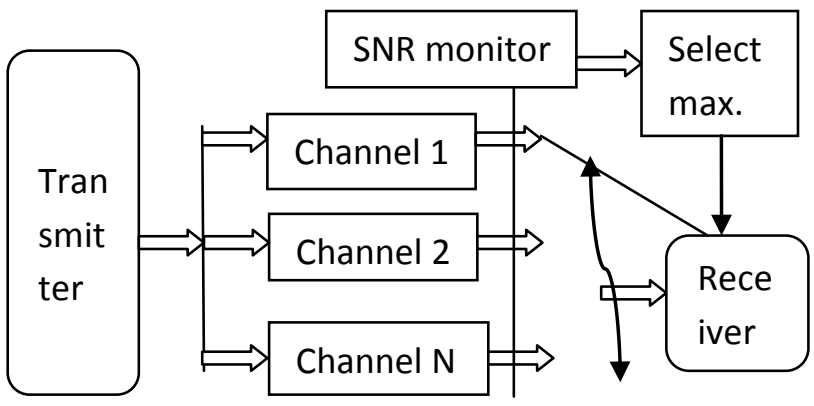

Fig 1: Selection Combining

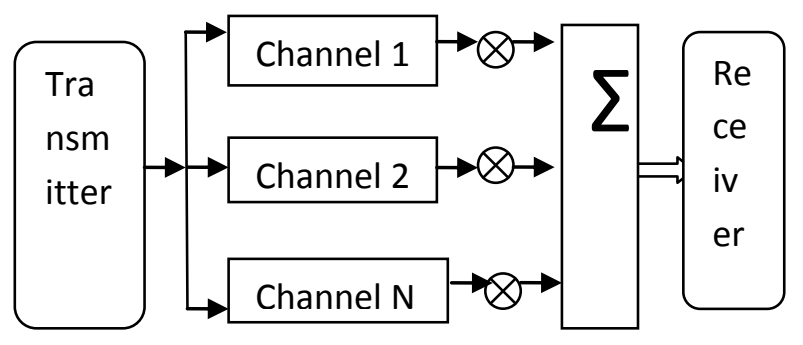

Fig 2: Maximal Ratio Combining 
In certain cases, it is not convenient to provide for the variable weighting capability required for true maximal ratio combining. In such cases, the branch weights are all set to unity, but the signals from each branch are co phased to provide equal gain combining diversity.

\subsection{Theoretical Analysis of Alamouti Code}

The Alamouti scheme [7] is a simple transmit diversity scheme suitable for two transmit antennas. At a given symbol period, two signals are simultaneously transmitted from the two antennas. The signal transmitted from antenna zero is denoted by $s_{0}$ and from antenna one by $s_{1}$. During the next symbol period signal $\left(-S_{1}^{*}\right)$ is transmitted from antenna zero, and signal $S_{0}^{*}$ is transmitted from antenna one where $*$ is the complex conjugate operation.

Table 1. The Encoding and Transmission sequence for the Two-Branch transmit Diversity Scheme

\begin{tabular}{|l|c|c|}
\hline Time & Antenna 0 & Antenna 1 \\
\hline $\mathrm{T}$ & $S_{0}$ & $S_{1}$ \\
\hline $\mathrm{t}+\mathrm{T}$ & $-S_{1}^{*}$ & $S_{0}^{*}$ \\
\hline
\end{tabular}

In Table 1, the encoding is done in space and time (space-time coding). The encoding, however, may also be done in space and frequency Instead of two adjacent symbol periods, two adjacent carriers may be used (space-frequency coding).

The channel at time $\mathrm{t}$ may be modeled by a complex multiplicative distortion $h_{0}(t)$ for transmit antenna zero and $h_{l}(t)$ for transmit antenna one. Assuming that fading is constant across two consecutive symbols, we can write

$$
\begin{aligned}
& h_{0}(t)=h_{0}(t+T)=h_{0}=\alpha_{0} e^{j \theta_{0}} \\
& h_{1}(t)=h_{1}(t+T)=h_{1}=\alpha_{1} e^{j \theta_{1}}
\end{aligned}
$$

Where $\mathrm{T}$ is the symbol duration. The received signals can then be expressed as

$r_{0}=r(t)=h_{0} s_{0}+h_{1} s_{1}+n_{0}$

$r_{1}=r(t+T)=-h_{0} s_{1}^{*}+h_{1} s_{0}^{*}+n_{1}$

Where $r_{0}$ and $r_{1}$ are the received signals at time $\mathrm{t}$ and $\mathrm{t}+\mathrm{T}$ and $n_{0}$ and $n_{1}$ are complex random variables representing receiver noise and interference.

\section{RESULTS AND DISCUSSIONS}

\subsection{Comparison between selection diversity scheme and maximal ratio combining scheme}

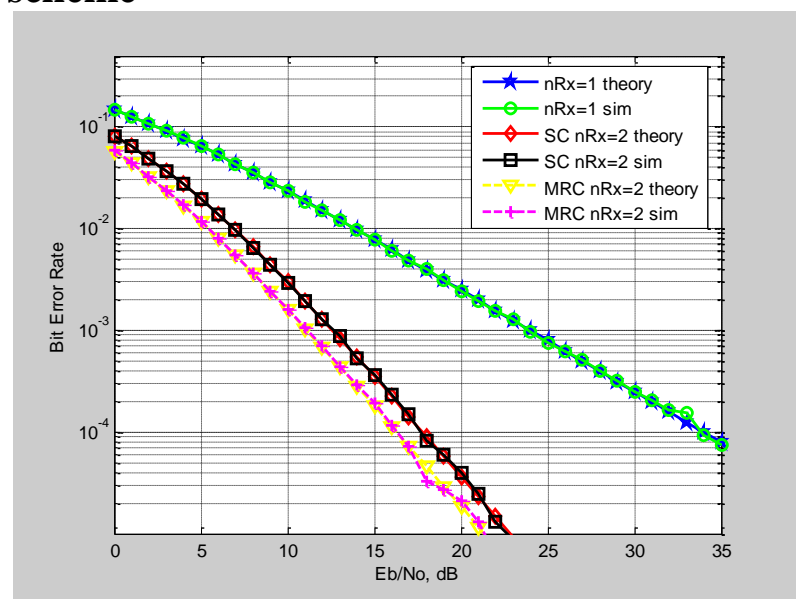

Fig 3: BER comparison of two branch selection diversity scheme with maximal ratio combining scheme for BPSK modulation in Rayleigh channel

Selection diversity offers an average improvement in the link margin without requiring additional transmitter power or sophisticated receiver circuitry it is easy to implement because all that is needed is a side monitoring station and an antenna switch at the receiver. However it is not an optimal diversity technique because it does not use all of the possible branches simultaneously.

When maximal ratio combining scheme is compared with selection diversity technique for two receive branch, it can be observed from above plot that maximal ratio combining scheme is better than selection diversity scheme.

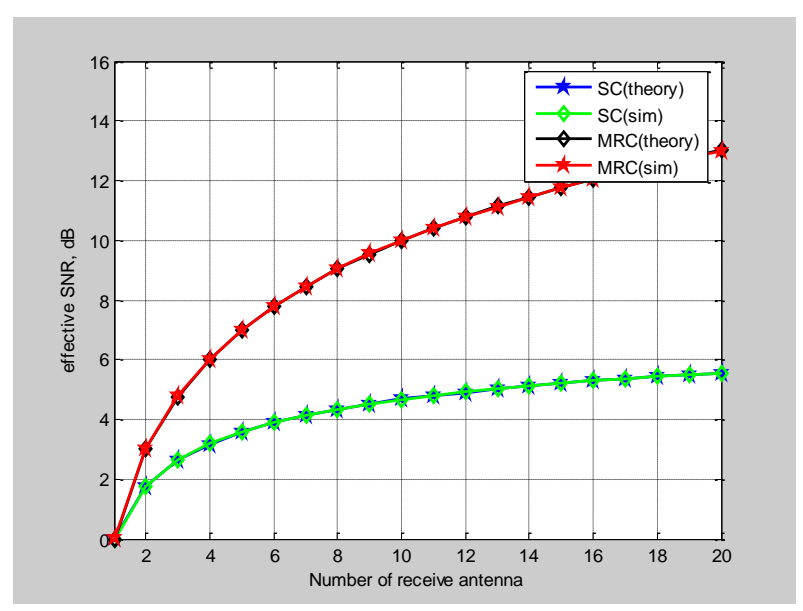

Fig 4: SNR improvement comparison of selection diversity scheme with maximal ratio combining scheme

As can be observed from above plot there is $4 \mathrm{~dB}$ performance improvement in case of maximal ratio combining scheme as compared to selection diversity scheme when number of receive antenna is six. 


\subsection{Comparison between equal gain combining scheme and maximal ratio combining scheme}

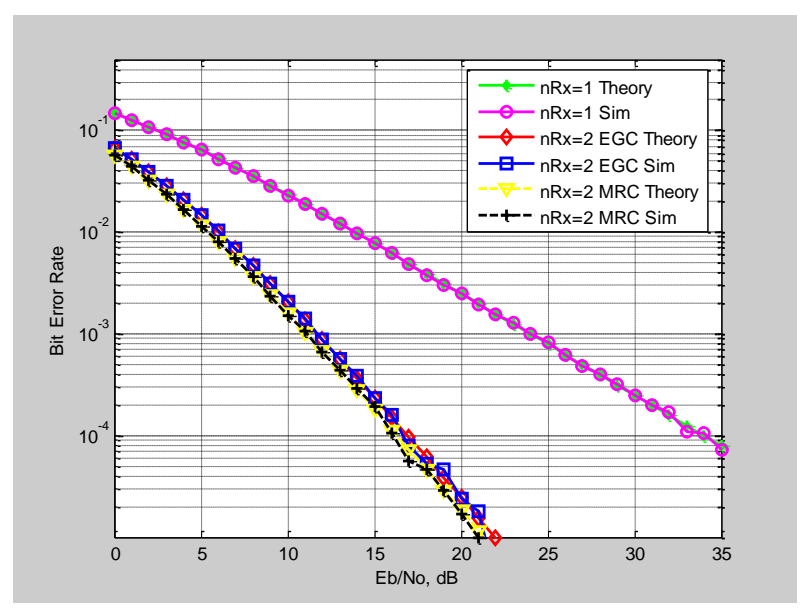

Fig 5: BER comparison of two branch equal gain combining scheme with maximal ratio combining scheme for BPSK modulation in Rayleigh channel

In certain cases, it is not convenient to provide for the variable weighting capability required for true maximal ratio combining. In such cases, the branch weights are all set to unity, but the signals from each branch are co phased to provide equal gain combining diversity.

When maximal ratio combining scheme is compared with equal gain combining technique for two receive branch, it can be observed from above plot that maximal ratio combining scheme is better than equal gain combining scheme.

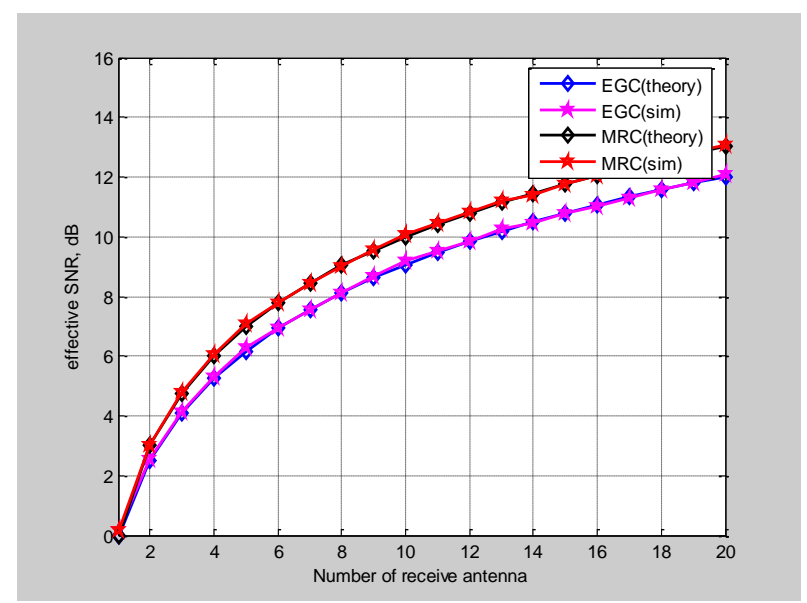

Fig 6: SNR improvement comparison of equal gain combining scheme with maximal ratio combining scheme

From above plot we can conclude that, improvement in the case of equal gain combining is comparable to that of maximal ratio combining. However there is slight improvement in SNR in case of maximal ratio combining scheme than that of equal gain combining scheme.

\subsection{Comparison between single antenna, maximal ratio combining and Alamouti dual antenna transmission scheme}

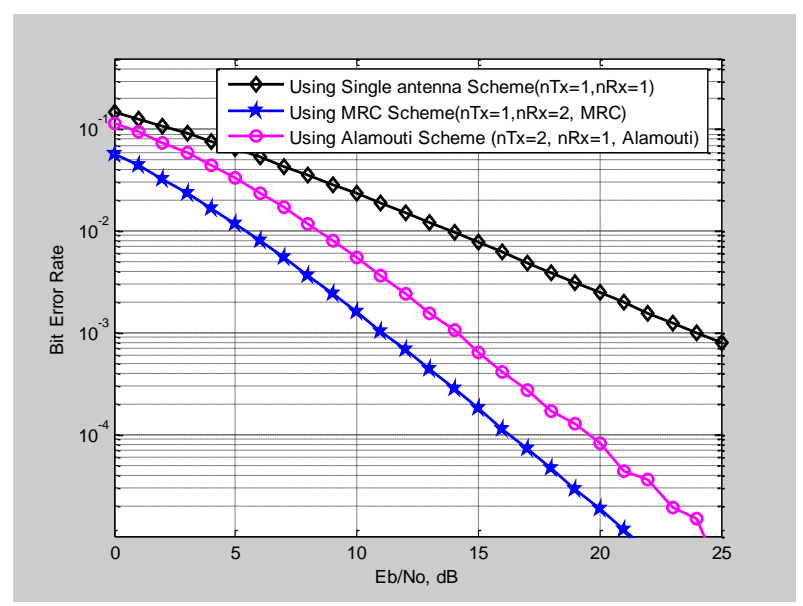

Fig 7: BER versus Eb/No for Aeronautical Telemetry using Single antenna, Maximal Ratio Combining \&

Alamouti dual Antenna transmission scheme

Figure 3 shows the bit error rate performance comparison between single antenna, maximal ratio combining scheme and Alamouti dual antenna transmission scheme using BPSK modulation for air-to-ground communication in aeronautical telemetry. These performance curves are simple reference illustration to show the performance improvement using Alamouti code as compared to single antenna scheme.

It is assumed that the receiver has perfect knowledge of the channel which is flat fading. The channel experienced between each transmit to the receive antenna is randomly varying in time, however, it is assumed to remain constant over two time slots.

Alamouti scheme requires the simultaneous transmission of two different symbols out of two antenna, hence the total transmit power in this scheme is twice as that used in MRC scheme. To make the comparison fair, we need to make the total transmit power from two antennas in Alamouti case to be equal to that of power transmitted from a single antenna in the MRC case. With this scaling, we can see that BER performance of two transmit, one receive Alamouti STBC case has a roughly $3 \mathrm{~dB}$ poorer performance than that of one transmit two receive antenna MRC case.

\section{Conclusion}

There is signal outage problem during testing of the latest aircraft, which cost a lot of money, during retesting. So possible causes of link loss and various fade mitigation techniques are analyzed. BER performance comparison as well as SNR comparison between selection diversity, equal gain combining (EGC) scheme, Alamouti dual antenna scheme and maximal ratio combining (MRC) is done, in order to determine best scheme for fade mitigation in aeronautical telemetry among all these four schemes.

It was found that maximal ratio combining (MRC) scheme is better than selection diversity equal gain combining (EGC) 
scheme and Alamouti dual antenna scheme BER performance wise.

\section{ACKNOWLEDGMENTS}

The authors would like to thank Defence Electronics Applications Laboratory (DEAL), and Dehradun Institute of Technology (DIT), Dehradun India, for encouraging us for this research work

\section{REFERENCES}

[1] Y. S. Meng and Y.H Lee, "Study of Shadowing Effect by Aircraft Maneuvering for Air-to-Ground Communication," AEU-International Journal of Electronics and Communications, Elsevier, 2011.

[2] R.M. Jha, S.A. Bokhari, V. Sudhakar and P.R. Mahapatra, "Ray analysis on a class of hybrid cylindrical aircraft wings," Electronics Letters, UK, vol. 24, pp. 2122, Jan. 1988.

[3] R.M. Jha, D.J. Edwards and W. Wiesbeck, "UTD analysis of the hyperbolic cylinders for applications to onboard aircraft antennas," IEEE $8^{\text {th }}$ Int. Conf. on Antennas and Propagation, ICAP'93, Edinburgh, UK, pp. 959-962, Mar. 30 - Apr. 2, 1993.

[4] T.S. Rappaport, "Wireless Communication: Principles and Practice", 2nd Edition, Prentice-Hall, India.

[5] D. G. Brennan, "Linear diversity combining techniques," Proc. IRE, vol. 47, pp.1075-1102, 1959.

[6] R.G. Vaughan and N. L. Scott, "Closely spaced monopoles for mobile communications," Radio Sci., vol. 28, pp. 1259-1266, 1993

[7] S. Alamouti, "A Simple Transmitter Diversity Technique for Wireless Communications", IEEE Journal on Selected Areas of Communications, Special Issue on Signal Processing for Wireless Communications, vol.16, no.8, pp.1451-1458, Oct 1998. 